Pacific

Journal of

Mathematics

\title{
SEPARATION OF RELATIVELY QUASICONVEX SUBGROUPS
}

JASON FoX MANNING AND EdUARdo MARTínEZ-PEDROZA 


\title{
SEPARATION OF RELATIVELY QUASICONVEX SUBGROUPS
}

\author{
JASON Fox MANNING AND EDUARdo MARTínEZ-PEDROZA
}

\begin{abstract}
We show that if all hyperbolic groups are residually finite, these statements follow: In relatively hyperbolic groups with peripheral structures consisting of finitely generated nilpotent subgroups, relatively quasiconvex subgroups are separable; geometrically finite subgroups of nonuniform lattices in rank one symmetric spaces are separable; Kleinian groups are subgroup separable. We also show that LERF for finite volume hyperbolic 3-manifolds would follow from LERF for closed hyperbolic 3-manifolds.

We prove these facts by reducing, via combination and filling theorems, the separability of a relatively quasiconvex subgroup of a relatively hyperbolic group $G$ to that of a quasiconvex subgroup of a hyperbolic quotient $\bar{G}$. A result of Agol, Groves, and Manning is then applied.
\end{abstract}

\section{Main results}

A subgroup $H$ of a group $G$ is called separable if for any $g \in G \backslash H$ there is a homomorphism $\pi$ onto a finite group such that $\pi(g) \notin \pi(H)$. A group is said to be residually finite if the trivial subgroup is separable, and subgroup separable or $L E R F$ if every finitely generated subgroup is separable. For example, Hall [1949] showed that free groups are LERF. It follows from a theorem of Mal'cev [1958] that polycyclic (and in particular finitely generated nilpotent) groups are LERF. A group is called slender if every subgroup is finitely generated. Polycyclic groups are also slender, by a result of Hirsch [1938].

Given a relatively hyperbolic group with peripheral structure consisting of LERF and slender subgroups, we study separability of relatively quasiconvex subgroups. This is connected, via filling constructions, to residual finiteness of hyperbolic groups. It is not known whether all word-hyperbolic groups are residually finite. Consequences of a positive or negative answer to this question have been explored by several authors; see for example [Agol et al. 2009; Kapovich and Wise 2000; Long and Niblo 1991; Minasyan 2006; Osin 2007; Wise 2002].

MSC2000: primary 20E26, 20F67; secondary 57M50.

Keywords: relatively hyperbolic group, quasiconvex subgroup, Kleinian group, residually finite,

subgroup separable, LERF, QCERF, geometrically finite, combination theorem, Dehn filling.

Manning was partially supported by NSF grant DMS-0804369. Martínez-Pedroza was funded as a Britton Postdoctoral Fellow at McMaster University, grant A4000. 
Theorem 1.1 [Agol et al. 2009]. If all hyperbolic groups are residually finite, then every quasiconvex subgroup of a hyperbolic group is separable.

We extend this result, answering a question in [Agol et al. 2009], as follows:

Theorem 1.2. Suppose that all hyperbolic groups are residually finite. If $G$ is a torsion free relatively hyperbolic group with peripheral structure consisting of subgroups that are LERF and slender, then any relatively quasiconvex subgroup of $G$ is separable.

This extension has some interesting corollaries. A pinched Hadamard manifold is a simply connected Riemannian manifold with pinched negative curvature. Bowditch [1995] gave several equivalent definitions of geometrical finiteness for discrete subgroups of the isometry group of a pinched Hadamard manifold, generalizing the notion of geometrical finiteness in Kleinian groups. The next theorem summarizes some useful facts about these groups. (Statement (1) can be found in [Farb 1998] or [Bowditch 1999]; statement (2) follows from the Margulis lemma; and statement (3) is [Hruska 2008, Corollary 1.3].)

Theorem 1.3. Let $X$ be a pinched Hadamard manifold and $G$ a geometrically finite subgroup of $\operatorname{Isom}(X)$.

(1) $G$ is hyperbolic relative to a collection of representatives of conjugacy classes of maximal parabolic subgroups.

(2) Maximal parabolic subgroups of $G$ are virtually nilpotent.

(3) A subgroup $H$ of $G$ is relatively quasiconvex if and only if $H$ is geometrically finite.

Rank one symmetric spaces are pinched Hadamard manifolds.

Corollary 1.4. Suppose that all hyperbolic groups are residually finite. Let $G$ be a discrete, geometrically finite subgroup of the isometry group of a rank one symmetric space. (For example, $G$ could be a lattice.) All the geometrically finite subgroups of $G$ are separable.

If the symmetric space is $\mathbb{\boxplus}^{3}$, more can be said - see Section 5 for the proof.

Corollary 1.5. If all hyperbolic groups are residually finite, then all finitely generated Kleinian groups are LERF.

We will prove Theorem 1.2 by combining one of Martínez-Pedroza's combination theorems [2009] with Theorem 1.1 and the Dehn filling technique of [Groves and Manning 2008; Osin 2007]. We next give a more detailed discussion.

Definition 1.6. A relatively quasiconvex subgroup $H$ of $G$ is called fully quasiconvex if for any subgroup $P \in \mathscr{P}$ and any $f \in G$, either $H \cap P^{f}$ is finite or $H \cap P^{f}$ is a finite index subgroup of $P^{f}$. (Here $P^{f}=f P f^{-1}$.) 
Using the work in [Martínez-Pedroza 2009], we show the following.

Theorem 1.7. Let $G$ be a group hyperbolic relative to a collection of slender and $L E R F$ subgroups. Suppose that $Q$ is a relatively quasiconvex subgroup of $G$ and $g$ $i$ an element of $G$ not in $Q$. Then there exists a fully quasiconvex subgroup $H$ that contains $Q$ and does not contain $g$.

Remark 1.8. When $G$ is a finite-volume hyperbolic 3-manifold group and $Q$ is the fundamental group of a quasifuchsian surface, Theorem 1.7 can be proved using geometric arguments like those in [Cooper and Long 1999; Cooper and Long 2001]. Such geometric arguments were applied (differently) to separability questions in [Agol et al. 2001]; see also [Wise 2006].

Using results of [Agol et al. 2009] and Theorem 1.7, we prove Theorem 1.9, a separation result of relatively quasiconvex subgroups by maps onto word hyperbolic groups. (Part of this theorem can be interpreted as saying that $G$ is "quasiconvex extended residually hyperbolic".)

Theorem 1.9. Let $G$ be a torsion free group hyperbolic relative to a collection of slender and LERF subgroups. For any relatively quasiconvex subgroup $Q$ of $G$ and any element $g \in G$ such that $g \notin Q$, there is a fully quasiconvex subgroup $H$ of $G$, and a surjective homomorphism $\pi: G \rightarrow \bar{G}$ such that

(1) $Q<H$,

(2) $\bar{G}$ is a word-hyperbolic group,

(3) $\pi(H)$ is a quasiconvex subgroup of $\bar{G}$, and

(4) $\pi(g) \notin \pi(H)$.

We can prove Theorem 1.2 from Theorem 1.9 as follows:

Proof of Theorem 1.2. Let $Q<G$ be relatively quasiconvex, and let $g \in G \backslash Q$. By Theorem 1.9, there is a fully quasiconvex $H<G$ containing $Q$ but not $g$, and a quotient $\pi: G \rightarrow K$ such that $\pi(g) \notin \pi(H), K$ is hyperbolic, and $\pi(K)$ is quasiconvex.

Assuming all hyperbolic groups are residually finite, Theorem 1.1 implies that there is a finite group $F$ and a quotient $\phi: K \rightarrow F$ such that $\phi(\pi(g)) \notin \phi(\pi(H))$. Since $\phi(\pi(H))$ contains $\phi(\pi(Q))$, the map $\phi \circ \pi$ serves to separate $g$ from $Q$.

Remark 1.10. The torsion-free hypotheses in Theorems 1.9 and 1.2 are not really necessary. We sketch the necessary changes to our argument in Appendix B. If one is primarily interested in Theorem 1.2 in the special case of virtually polycyclic peripheral subgroups, we have a simple argument, pointed out to us by the referee:

Let $G$ be a group hyperbolic relative to a collection $\mathscr{P}=\left\{P_{1}, \ldots, P_{m}\right\}$ of virtually polycyclic subgroups. An easy argument shows that each $P_{i}$ contains a finite index normal subgroup that is torsion free. Moreover, $G$ contains only finitely many finite 
order nonparabolic elements, up to conjugacy [Osin 2006, Theorem 4.2]. It then follows from Osin's version of the relatively hyperbolic Dehn filling theorem that there is a filling $G \stackrel{\pi}{\longrightarrow} G\left(N_{1}^{\prime}, \ldots, N_{m}^{\prime}\right)$ such that $G\left(N_{1}^{\prime}, \ldots, N_{m}^{\prime}\right)$ is hyperbolic and no nontrivial torsion element of $G$ is in the kernel of $\pi$. Assuming hyperbolic groups are residually finite, the group $G\left(N_{1}^{\prime}, \ldots, N_{m}^{\prime}\right)$ has a torsion-free subgroup $S$ of finite index. The preimage $G_{0}=\pi^{-1}(S)$ is a torsion-free finite index subgroup of $G$. Again under the assumption that hyperbolic groups are residually finite, Theorem 1.2 implies that $G_{0}$ is QCERF, and so, applying Corollary 2.4 below, $G$ must also be QCERF.

The paper is organized as follows. In Section 2.5, we define relatively hyperbolic groups in a way that suits this paper and is equivalent to the more standard definitions. In Section 3, we recall a combination theorem for relatively quasiconvex subgroups from [Martínez-Pedroza 2009] and prove Theorem 1.7. In Section 4, we recall some definitions and results on fillings of relatively hyperbolic groups and prove Theorem 1.9. In Section 5, we give two applications to separability questions on hyperbolic 3-manifolds: Corollary 1.5 and Proposition 5.3. In Appendix A, we prove a result we need on the equivalence of various definitions of relative quasiconvexity, and in Appendix B, we sketch how to prove our results in the presence of torsion.

\section{Preliminaries}

2.1. Separability. Let $G$ be a group. Recall that the profinite topology on $G$ is the smallest topology on $G$ in which all finite index subgroups and their cosets are closed. The group $G$ is residually finite if and only if this topology is Hausdorff. A subgroup $H$ is separable if and only if it is a closed subset of $G$ in this topology.

Given a subgroup $G_{0}<G$, one can ask whether the profinite topology on $G_{0}$ coincides with the topology induced by the profinite topology on $G$. In general, the topologies are quite different, but in case $G_{0}$ is finite index, the topologies coincide.

Lemma 2.2. Let $G_{0}<G$ be a finite index subgroup, and let $C_{0} \subseteq G_{0}$. The following conditions are equivalent:

(1) $C_{0}$ is closed in the profinite topology on $G_{0}$.

(2) $C_{0}$ is closed in the profinite topology on $G$.

(3) $C_{0}=C \cap G_{0}$ for some set $C$ that is closed in the profinite topology on $G$.

Proof. Suppose $C_{0}$ is closed in $G_{0}$. Since the finite index subgroups of $G_{0}$ and their cosets generate the topology on $G_{0}$, we can write $C_{0}$ as a finite union of arbitrary 
intersections of cosets

$$
C_{0}=\bigcup_{i=1}^{n} \bigcap_{i \in I_{j}} g_{i} K_{i},
$$

where every $K_{i}$ is a finite index subgroup of $G_{0}$. But since $G_{0}$ is finite index in $G$, each of the $K_{i}$ appearing in Equation (2-1) is also finite index in $G$. Thus $C_{0}$ is a finite union of intersections of closed sets in the profinite topology on $G$, so $C_{0}$ is closed in $G$. Thus condition (1) implies condition (2).

Trivially, condition (2) implies condition (3). To see that condition (3) implies condition (1), we first establish the following.

Claim 2.3. If $g \in G$, and $K<G$ is finite index, then $g K \cap G_{0}$ is closed in the profinite topology on $G_{0}$.

Proof. Let $K_{0}=K \cap G_{0}$. Since $K_{0}$ is finite index in $K$, the subgroup $K$ is a finite union of cosets $K=\bigcup_{i=1}^{p} g_{i} K_{0}$, and so $g K=\bigcup_{i=1}^{p} g g_{i} K_{0}$ is as well. Since a coset of $K_{0}$ in $G$ either lies inside $G_{0}$ or in its complement, $g K \cap G_{0}$ is a finite union of cosets of $K_{0}$ in $G_{0}$. Since $K_{0}$ is finite index in $G_{0}$, these cosets are closed in the profinite topology on $G_{0}$ and therefore $g K \cap G_{0}$ is closed as well.

Suppose then that $C_{0}=C \cap G_{0}$ for $C$ closed in $G$. Then $C=\bigcup_{i=1}^{n} \bigcap_{i \in I_{j}} g_{i} K_{i}$, where now the $K_{i}$ are arbitrary finite index subgroups of $G$, and the $g_{i}$ are arbitrary elements of $G$. We thus have

$$
C_{0}=C \cap G_{0}=\bigcup_{i=1}^{n} \bigcap_{i \in I_{j}}\left(g_{i} K_{i}\right) \cap G_{0} .
$$

By the claim, every $\left(g_{i} K_{i}\right) \cap G_{0}$ appearing here is closed in $G_{0}$, and so $C_{0}$ is also closed in the profinite topology on $G_{0}$.

Corollary 2.4. Let $H<G$ be a pair of groups, and let $G_{0}$ be a finite index subgroup of $G$. Let $H_{0}=H \cap G_{0}$. The subgroup $H$ is separable in $G$ if and only if $H_{0}$ is separable in $G_{0}$.

Another easy corollary is that LERFness and QCERFness are commensurability invariants.

2.5. Relative hyperbolicity. Several authors have studied relative hyperbolicity, using different but equivalent definitions. The definition we use here is based on [Osin 2006]. Let $G$ be a group, let $\mathscr{P}$ denote a collection of subgroups $\left\{P_{1}, \ldots, P_{m}\right\}$, and let $S$ be a finite generating set assumed to be symmetric, that is, $S=S^{-1}$. Denote by $\Gamma(G, \mathscr{P}, S)$ the Cayley graph of $G$ with respect to the generating set $S \cup \bigcup \mathscr{P}$. Given a path $p$ between vertices in $\Gamma(G, \mathscr{P}, S)$, we will call the initial vertex $p_{-}$and the terminal vertex $p_{+}$. The path $p$ determines a word $\operatorname{Label}(p)$ in the alphabet $S \cup \bigcup \mathscr{P}$; this word represents an element $g$ such that $p_{+}=p_{-} g$. 
Definition 2.6 (weak relative hyperbolicity). The pair $(G, \mathscr{P})$ is weakly relatively hyperbolic if there is an integer $\delta \geq 0$ such that $\Gamma(G, \mathscr{P}, S)$ is $\delta$-hyperbolic. We may also say that $G$ is weakly hyperbolic relative to $\mathscr{P}$.

Definition 2.7 [Osin 2006]. Let $q$ be a combinatorial path in the Cayley graph $\Gamma(G, \mathscr{P}, S)$. Subpaths of $q$ with at least one edge are called nontrivial. For $P_{i} \in \mathscr{P}$, a $P_{i}$-component of $q$ is a maximal nontrivial subpath $s$ of $q$ with $\operatorname{Label}(s)$ a word in the alphabet $P_{i}$. When we don't need to specify the index $i$, we will refer to $P_{i}$-components as $\mathscr{P}$-components.

Two $\mathscr{P}$-components $s_{1}$ and $s_{2}$ are connected if their vertices belong to the same left coset of $P_{i}$ for some $i$. A $\mathscr{P}$-component $s$ of $q$ is isolated if it is not connected to a different $\mathscr{P}$-component of $q$. The path $q$ is without backtracking if every $\mathscr{P}$-component of $q$ is isolated.

A vertex $v$ of $q$ is called phase if it is not an interior vertex of a $\mathscr{P}$-component $s$ of $q$. Let $p$ and $q$ be paths between vertices in $\Gamma(G, \mathscr{P}, S)$. The paths $p$ and $q$ are $k$-similar if

$$
\max \left\{\operatorname{dist}_{S}\left(p_{-}, q_{-}\right), \operatorname{dist}_{S}\left(p_{+}, q_{+}\right)\right\} \leq k,
$$

where dist ${ }_{S}$ is the metric induced by the finite generating set $S$ (as opposed to the metric in $\Gamma(G, \mathscr{P}, S))$.

Remark 2.8. A geodesic path $q$ in $\Gamma(G, \mathscr{P}, S)$ is without backtracking, all $\mathscr{P}$ components of $q$ consist of a single edge, and all vertices of $q$ are phase.

Definition 2.9 (bounded coset penetration, or BCP). The pair $(G, \mathscr{P})$ satisfies the $B C P$ property if for any $\lambda \geq 1, c \geq 0$, and $k \geq 0$, there is an integer $\epsilon(\lambda, c, k)>0$ such that if $p$ and $q$ are any two $k$-similar $(\lambda, c)$-quasigeodesics in $\Gamma(G, \mathscr{P}, S)$ without backtracking, these conditions hold:

(i) The sets of phase vertices of $p$ and $q$ are contained in the closed $\epsilon(\lambda, c, k)$ neighborhoods of each other, with respect to the metric dist ${ }_{S}$.

(ii) If $s$ is any $\mathscr{P}$-component of $p$ such that $\operatorname{dist}_{S}\left(s_{-}, s_{+}\right)>\epsilon(\lambda, c, k)$, then there exists a $\mathscr{P}$-component $t$ of $q$ that is connected to $s$.

(iii) If $s$ and $t$ are connected $\mathscr{P}$-components of $p$ and $q$ respectively, then

$$
\max \left\{\operatorname{dist}_{S}\left(s_{-}, t_{-}\right), \operatorname{dist}_{S}\left(s_{+}, t_{+}\right)\right\} \leq \epsilon(\lambda, c, k) .
$$

Remark 2.10. Our definition of the BCP property corresponds to the conclusion of [Osin 2006, Theorem 3.23].

Definition 2.11 (relative hyperbolicity). The pair $(G, \mathscr{P})$ is relatively hyperbolic if the group $G$ is weakly hyperbolic relative to $\mathscr{P}$ and the pair $(G, \mathscr{P})$ satisfies the BCP property. If $(G, \mathscr{P})$ is relatively hyperbolic, then we say $G$ is hyperbolic relative to $\mathscr{P}$; if there is no ambiguity, we just say that $G$ is relatively hyperbolic. 
Remark 2.12. Definition 2.11 is equivalent to [Osin 2006, Definition 2.35] for finitely generated groups: To see that Osin's definition implies Definition 2.11, apply Osin's Theorem 3.23; to see the converse, apply Osin's Lemma 7.9 and Theorem 7.10. For the equivalence of Osin's definition and the various other definitions of relative hyperbolicity, see [Hruska 2008] and the references therein.

The definition of relative hyperbolicity is independent of finite generating set $S$.

\section{Combination of parabolic and quasiconvex subgroups}

In this section, $G$ will be hyperbolic relative to a finite collection of subgroups $\mathscr{P}=$ $\left\{P_{1}, \ldots, P_{m}\right\}$, and $S$ will be a finite generating set for $G$. Denote by $\Gamma(G, \mathscr{P}, S)$ the Cayley graph of $G$ with respect to the generating set $S \cup \bigcup \mathscr{P}$.

\subsection{Parabolic and quasiconvex subgroups.}

Definition 3.2. The peripheral subgroups of $G$ are the elements of $\mathscr{P}$. A subgroup of $G$ is called parabolic if it can be conjugated into a peripheral subgroup.

Proposition 3.3 [Osin 2006, Proposition 2.36]. Let $P_{i}, P_{j} \in \mathscr{P}$, and let $g \in G$. Unless $i=j$ and $g \in P_{i}$, the intersection $P_{i}^{g} \cap P_{j}$ is finite.

In particular, if $Q$ is a subgroup of $G$, then any infinite maximal parabolic subgroup of $Q$ is of the form $Q \cap P_{i}^{f}$ for some $f \in Q$ and some $P_{i} \in \mathscr{P}$.

Definition 3.4 [Osin 2006, Definition 4.9]. A subgroup $Q$ of $G$ is called quasiconvex relative to $\mathscr{P}$ (or simply relatively quasiconvex when the collection $\mathscr{P}$ is fixed) if there exists a constant $\sigma \geq 0$ such that the following holds: Let $f, g \in Q$, and let $p$ be an arbitrary geodesic path from $f$ to $g$ in the Cayley graph $\Gamma(G, \mathscr{P}, S)$. For any vertex $v \in p$, there exists a vertex $w \in Q$ such that $\operatorname{dist}_{S}(v, w) \leq \sigma$, where dist $_{S}$ is the word metric induced by $S$.

Remark 3.5. For more on different definitions of relative quasiconvexity in the literature, see Appendix A.

Theorem 3.6 [Hruska 2008, Theorem 9.1]. Let $Q$ be a finitely generated relatively quasiconvex subgroup of $G$. The number of infinite maximal parabolic subgroups of $Q$ up to conjugacy in $Q$ is finite. Also, if 0 is a set of representatives of these conjugacy classes, then $Q$ is hyperbolic relative to 0 .

Remark 3.7. Hruska [2008] uses an extended definition of relative hyperbolicity that includes some countable but nonfinitely generated groups. Using this extended definition, the assumption of finite generation in Theorem 3.6 is superfluous.

In case all the peripheral subgroups are slender, relatively quasiconvex subgroups are necessarily finitely generated; see [Hruska 2008, Corollary 9.2]. 
3.8. Combination of quasiconvex subgroups. For $g \in G$, we denote by $|g|_{S}$ the distance from $g$ to the identity element in the word metric induced by $S$.

Theorem 3.9 [Martínez-Pedroza 2009, Theorem 1.1]. Let $Q$ be a relatively quasiconvex subgroup of $G$, and let $P$ be a maximal parabolic subgroup of $G$. Suppose that $P^{f}=P_{i}$ for some $P_{i} \in \mathscr{P}$ and $f \in G$.

There are constants $C=C(Q, P) \geq 0$ and $c=c(Q, P) \geq 0$ with the following property. Suppose $D \geq C$, and $R$ is a subgroup of $P$ such that $P \cap Q<R$ and $|g|_{S}>D$ for every element $g \in R \backslash Q$. It follows that

(1) the subgroup $H=\langle Q \cup R\rangle$ is relatively quasiconvex and the natural map $Q * Q \cap R R \rightarrow H$ is an isomorphism;

(2) every parabolic subgroup of $H$ is conjugate in $H$ to a parabolic subgroup of $Q$ or $R$;

(3) for any $g \in H$, either $g$ belongs to $Q$ or any geodesic from 1 to $g$ in the relative Cayley graph $\Gamma(G, \mathscr{P}, S)$ has at least one $P_{i}$-component $t$ such that $|t|_{S}>D-c$.

Proof. Conclusions (1) and (2) rephrase [Martínez-Pedroza 2009, Theorem 1.1]. The proof of conclusion (3) is divided into two cases: $P \in \mathscr{P}$ and $P \notin \mathscr{P}$.

Case 1: $P \in \mathscr{P}$. We summarize part of the argument for [Martínez-Pedroza 2009, Theorem 1.1] for conclusions (1) and (2); we then explain how conclusion (3) follows in this case.

Let $g \in Q * Q \cap R \backslash Q$. The element $g$ has a normal form

$$
g=g_{1} h_{1} \cdots g_{k} h_{k},
$$

where $g_{j} \in Q \backslash Q \cap R$ for $1<j \leq k, h_{j} \in R \backslash Q \cap R$ for $1 \leq j<k$, either $g_{1}=1$ or $g_{1} \in Q \backslash Q \cap R$, and either $h_{k}=1$ or $h_{k} \in R \backslash Q \cap R$. We use the normal form to produce a path $o$ in $\Gamma(G, \mathscr{P}, S)$ from 1 to the image of $g$ by the natural map $Q * Q \cap R \quad R \rightarrow H$ as follows. For each $j$ between 1 and $k$, let $u_{j}$ be a geodesic path in $\Gamma(G, \mathscr{P}, S)$ from $g_{1} h_{1} \cdots h_{j}$ to $g_{1} h_{1} \cdots h_{j} g_{j}$ (so that $\operatorname{Label}\left(u_{j}\right)$ represents $g_{j}$ ). Similarly, let $v_{j}$ be a geodesic path from $g_{1} h_{1} \cdots g_{j-1}$ to $g_{1} h_{1} \cdots g_{j-1} h_{j}$ (so that $\operatorname{Label}\left(v_{j}\right)$ represents $\left.h_{j}\right)$. A path $o$ from 1 to $g$ in $\Gamma(G, \mathscr{P}, S)$ is given by

$$
o=u_{1} v_{1} \ldots u_{k} v_{k} .
$$

See Figure 1.

Each subsegment $v_{j}$ is part of a $\mathscr{P}$-component $t_{j}$ of the path $o$. Let $D$ be as in the hypothesis of the theorem. The penultimate inequality in the proof of Claim 2 in the proof of [Martínez-Pedroza 2009, Lemma 5.1] is

$$
\left|t_{j}\right|_{S}=\operatorname{dist}_{S}\left(\left(t_{j}\right)_{-},\left(t_{j}\right)_{+}\right)>D-2 M(P, Q, \sigma),
$$




\section{$\Gamma(G, \mathscr{P}, S)$}

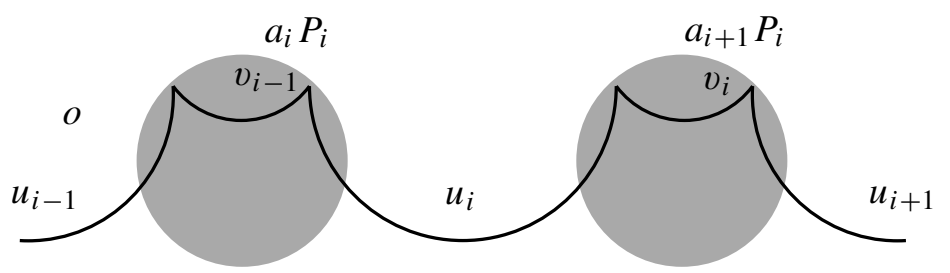

Figure 1. Part of the polygonal path $o$ in $\Gamma(G, \mathscr{P}, S)$. For each $i$, the $P_{i}$-component of $o$ containing the subsegment $v_{j}$ is long with respect to the $S$-metric. This implies that the path $o$ is a quasigeodesic with different endpoints.

where $\sigma$ is the quasiconvexity constant for $Q$ and $M(P, Q, \sigma)$ is the constant provided by [Martínez-Pedroza 2009, Lemma 4.2] for the subgroups $Q, P$, and the constant $\sigma$. Let $\eta$ be the constant from [Martínez-Pedroza 2009, Proposition 3.1]. If $D-2 M(P, Q, \sigma)>\eta$, then $o$ is a $(\lambda, 0)$-quasigeodesic with distinct endpoints. Let $C=\eta+2 M(P, Q, \sigma)$.

It follows from the argument just sketched that if $D>C$, then the natural map $Q *{ }_{Q \cap R} R \rightarrow H$ is an isomorphism. It can further be shown that $H$ is a relatively quasiconvex subgroup and that the parabolic subgroups of $H$ are conjugate into $Q$ or $R$ by elements of $H$. See [Martínez-Pedroza 2009, Lemmas 5.2 and 5.3] for details. In other words, conclusions (1) and (2) hold for $P \in \mathscr{P}$ and $C$ as above.

If $g \in H \backslash Q$ and $p$ is a geodesic from 1 to $g$ in $\Gamma(G, \mathscr{P}, S)$, then the $(\lambda, 0)$ quasigeodesic $o$ and the geodesic $p$ are 0 -similar. Since $o$ has a $\mathscr{P}$-component of $S$-length at least $D-2 M(P, Q, \sigma)$, the BCP property of Definition 2.9 implies that $p$ has a $\mathscr{P}$-component of $S$-length at least $D-2 M(P, Q, \sigma)-2 \epsilon(0, \lambda, 0)$. In the case that $f=1$ and $P \in \mathscr{P}$, we have verified conclusion (3) of the theorem for $c$ equal to

$$
c(Q, P)=2 M(P, Q, \sigma)+2 \epsilon(0, \lambda, 0) .
$$

Case 2: $P \notin \mathscr{P}$, but $P^{f} \in \mathscr{P}$ for some $f \in G \backslash P$. Since $P^{f} \in \mathscr{P}$ and $Q^{f}$ is relatively quasiconvex, by [Martínez-Pedroza 2009, Theorem 1.1] and Case 1, all three conclusions of Theorem 3.9 hold for $Q^{f}$ and $P^{f}$ and some constants $C^{\prime}=C\left(Q^{f}, P^{f}\right)>0$ and $c^{\prime}=c\left(Q^{f}, P^{f}\right)>0$. Define

$$
C=C^{\prime}+2|f|_{S}+3 \epsilon\left(1,0,|f|_{S}\right) \text { and } c=c^{\prime}+2|f|_{S}+2 \epsilon\left(1,0,|f|_{S}\right),
$$

where $\epsilon\left(1,0,|f|_{S}\right)$ is the BCP constant from Definition 2.9. Now we show that the theorem holds for the subgroups $P$ and $Q$, and the constants $C$ and $c$. Let $R$ be a subgroup of $Q$ satisfying the hypothesis of the theorem for a constant $D>C$. 
If $r \in R \backslash Q$, then $|r|_{S} \geq D$, by hypothesis. It follows that $\left|r^{f}\right|_{S} \geq D-2|f|_{S} \geq C^{\prime}$. Therefore:

- The subgroup $H^{f}=\left\langle Q^{f} \cup R^{f}\right\rangle$ is relatively quasiconvex and the natural map $Q^{f} *_{(Q \cap R)^{f}} R^{f} \rightarrow H^{f}$ is an isomorphism. Since relative quasiconvexity is preserved by conjugation, $H=\langle Q \cup R\rangle$ is relatively quasiconvex. Obviously the map $Q * Q \cap R R \rightarrow H$ is also an isomorphism. In other words, conclusion (1) holds for $Q$ and $P$ and the constant $C$.

- Every parabolic subgroup of $H^{f}$ is conjugate in $H^{f}$ to a parabolic subgroup of $Q^{f}$ or $R^{f}$. Parabolicity is preserved under conjugation, so conclusion (2) holds for the subgroups $Q, R$, and $H$, and the constant $C$.

- For any $h \in H^{f}$, either $h \in Q^{f}$ or any geodesic from 1 to $h$ in the relative Cayley graph $\Gamma(G, \mathscr{P}, S)$ has at least one $P_{i}$-component $t$ that satisfies $|t|_{S}>D-2|f|_{S}-c^{\prime}$.

It remains to see why conclusion (3) holds with the chosen constant $c$. Let $g \in H \backslash Q$ and let $p$ be a geodesic from 1 to $g$ in $\Gamma(G, \mathscr{P}, S)$. We must show that $p$ has a $P_{i}$-component of $S$-length at least $D-c$. Let $q$ be a geodesic from 1 to $f g f^{-1}$. Since $f g f^{-1}$ belongs to $H^{f} \backslash Q^{f}$, the geodesic $q$ has a $P_{i}$-component $u$ of $S$-length at least $D-2|f|_{S}-c^{\prime}$. Let $r$ be the geodesic starting at $f$, with the same label as $p$. Thus $r$ joins $f$ to $f g$, and the geodesics $q$ and $r$ are $|f|{ }_{S}$-similar; see Figure 2.

By the BCP property, $r$ has a $P_{i}$-component $v$ of $S$-length at least

$$
D-2|f|_{S}-c^{\prime}-2 \epsilon\left(1,0,|f|_{S}\right)=D-c .
$$

Since $p=f^{-1} r$ and $r$ have the same labels, it follows that $p$ has a $P_{i}$-component $w$ of $S$-length at least $D-c$.

Corollary 3.10 [Martínez-Pedroza 2009, Lemma 5.4]. Suppose that $G, Q, P$, and $R$ are as assumed in Theorem 3.9 and that $Q \cap P$ is a proper subgroup of $R$.

If $\left\{K_{1}, \ldots, K_{n}\right\}$ is a set of representatives of the maximal infinite parabolic subgroups of $Q$ up to conjugacy in $Q$, such that $K_{1}=P \cap Q$, then $\left\{R, K_{2}, \ldots, K_{n}\right\}$ is a set of representatives of the maximal parabolic subgroups of $H$ up to conjugacy in $H$.

Proof. By Theorem 3.9(2), a maximal parabolic subgroup of $H$ is conjugate to $R$ or $K_{i}$ for some $i=2, \ldots n$. Hence $\left\{R, K_{2}, \ldots, K_{n}\right\}$ is a set of representatives of maximal parabolic subgroups. That all these subgroups are different up to conjugacy follows from the algebraic structure of $H$ as an amalgamated product. In particular, since $K_{i}$ and $K_{j}$ are not conjugate in $Q$, they are not conjugate in $Q * Q \cap R$. The subgroup $R<Q * Q \cap R R$ is not conjugate to a subgroup of $Q$ since $Q \cap R$ is a proper subgroup of $R$. 


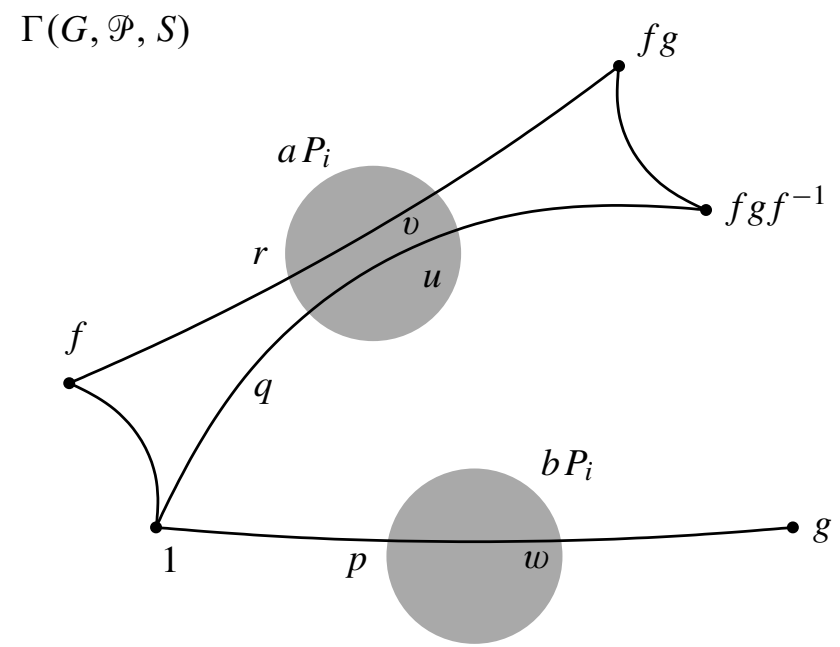

Figure 2. The geodesics $q$ and $r$ are $|f|_{S}$-similar and $q$ contains a large $\mathscr{P}$-component $u$. By the BCP-property, $r$ has a large $\mathscr{P}$ component $v$. Since $p$ and $r$ have the same word-label, $p$ has a large $\mathscr{P}$-component $w$.

Proof of Theorem 1.7. Suppose that every subgroup in $\mathscr{P}$ is LERF and slender. Let $Q$ be a relatively quasiconvex subgroup of $G$, and let $g$ be an element of $G$ not in $Q$. Let $\left\{K_{1}, \ldots, K_{n}\right\}$ be a set of representatives of maximal infinite parabolic subgroups of $Q$ up to conjugacy in $Q$; such a set exists by Theorem 3.6. By Proposition 3.3, for each $K_{i}$ there is a peripheral subgroup $P_{i} \in \mathscr{P}$ and $f_{i} \in G$ such that $K_{i}<P_{i}^{f_{i}}$.

We will construct an ascending sequence of relatively quasiconvex subgroups

$$
Q=Q_{0}<Q_{1}<\cdots<Q_{n}=H
$$

such that for each $k \in\{1, \ldots, n\}$ the following properties hold.

(i) For $0 \leq j \leq k$, the subgroup $Q_{k} \cap P_{j}^{f_{j}}$ is finite index in $P_{j}^{f_{j}}$.

(ii) $\left\{Q_{k} \cap P_{1}^{f_{1}}, \ldots, Q_{k} \cap P_{k}^{f_{k}}, K_{k+1}, \ldots, K_{n}\right\}$ is a set of representatives of the maximal parabolic subgroups of $Q_{k}$ up to conjugation in $Q_{k}$.

(iii) $g \notin Q_{k}$.

It will follow that $H=Q_{n}$ is a fully quasiconvex subgroup that contains $Q$ and does not contain the element $g$.

Choose a geodesic $p$ from 1 to $g$ in the relative Cayley graph $\Gamma(G, \mathscr{P}, S)$. Let $L$ be an upper bound for the $S$-length of the $P_{k}$-components of the path $p$. 
We now show how to construct $Q_{k}$, assuming that $Q_{k-1}$ is already constructed. Let $C$ and $c$ be the constants provided by Theorem 3.9 for the subgroups $Q_{k-1}$ and $P_{k}^{f_{k}}$. Since $P_{k}^{f_{k}}$ is slender, $K_{k}=Q_{k-1} \cap P_{k}^{f_{k}}$ is finitely generated. Define a finite set $F \subset P_{k}^{f_{k}} \backslash K_{k}$ by

$$
F= \begin{cases}\left\{\left.p \in P_{k}^{f_{k}} \backslash K_{k}|| p\right|_{S} \leq L+C+c\right\} \cup\{g\} & \text { if } g \in P_{k}^{f_{k}}, \\ \left\{\left.p \in P_{k}^{f_{k}} \backslash K_{k}|| p\right|_{S} \leq L+C+c\right\} & \text { otherwise. }\end{cases}
$$

Because $P_{k}^{f_{k}} \cong P_{k}$ is LERF, we may find a finite index subgroup $R_{k}$ of $P_{k}^{f_{k}}$ such that $K_{k}<R_{k}$ and $f \notin R_{k}$ for all $f \in F$. In particular, $|h|_{S}>L+C+c$ for any $h \in R_{k} \backslash Q_{k-1}$. Let $Q_{k}=\left\langle Q_{k-1} \cup R_{k}\right\rangle$. Note that the hypotheses of the combination Theorem 3.9 (and hence those of Corollary 3.10) are satisfied for the relatively quasiconvex subgroup $Q_{k-1}$ and the parabolic subgroup $R_{k}$.

We now verify properties (i)-(iii) for the subgroup $Q_{k}$ just constructed. The first follows because $Q_{k}$ contains $Q_{j} \cap P_{j}^{f_{j}}$ for each $j$ between 1 and $k-1$, and also contains $R_{k}$. Corollary 3.10 implies that property (ii) is satisfied. By Theorem 3.9(3), any geodesic in the Cayley graph $\Gamma(G, S \cup \cup \mathscr{P})$ from 1 to an element of $Q_{k}$ that is not in $Q_{k-1} \cup R_{k}$ has a $P_{k}$-component of $S$-length greater than $L+C$; it follows that the element $g$ does not belong to $Q_{k}$, and so property (iii) is also satisfied. This concludes the construction of the group $Q_{k}$, and the theorem follows by taking $H=Q_{n}$.

\section{Fillings of relatively hyperbolic groups}

Suppose $G$ is torsion free and hyperbolic relative to a collection of subgroups $\mathscr{P}=\left\{P_{1}, \ldots, P_{m}\right\}$, and let $S \subset G$ be a finite generating set of $G$. Suppose that $S \cap P_{i}$ is a generating set of $P_{i}$ for each $i$.

Definition 4.1. A filling of $G$ is determined by a collection of subgroups $\left\{N_{i}\right\}_{i=1}^{m}$ such that $N_{i}$ is a normal subgroup of $P_{i}$ for each $i$; these subgroups are called filling kernels. The quotient of $G$ by the normal subgroup generated by $\bigcup_{i=1}^{m} N_{i}$ is denoted by $G\left(N_{1}, \ldots, N_{m}\right)$.

The following result is due (in the present setting) independently to Groves and Manning [2008, Theorem 7.2 and Corollary 9.7] and to Osin [2007, Theorem 1.1]. (Osin actually proves a more general result, in which $G$ may have torsion.)

Theorem 4.2 [Groves and Manning 2008; Osin 2007]. Let $F$ be a finite subset of $G$. There exists a constant $B$ depending on $G, \mathscr{P}, S$, and $F$ with the following property. If a collection of filling kernels $\left\{N_{i}\right\}_{i=1}^{m}$ satisfies $|f|_{S}>B$ for every nontrivial $f \in \bigcup_{i} N_{i}$, then

(1) the natural map $\imath_{i}: P_{i} / N_{i} \rightarrow G\left(N_{1}, \ldots, N_{m}\right)$ is injective,

(2) $G\left(N_{1}, \ldots, N_{m}\right)$ is hyperbolic relative to $\left\{l_{i}\left(P_{i} / N_{i}\right)\right\}_{i=1}^{m}$, and 
(3) the projection $G \rightarrow G\left(N_{1}, \ldots, N_{m}\right)$ is injective on $F$.

4.3. Fillings and quasiconvex subgroups. Let $H$ be a relatively quasiconvex subgroup of $G$. A filling $G \rightarrow G\left(N_{1}, \ldots, N_{m}\right)$ is an $H$-filling if $N_{i}^{g} \subset P_{i}^{g} \cap H$ whenever $H \cap P_{i}^{g}$ is nontrivial.

Theorem 4.4 [Agol et al. 2009, Propositions 4.3 and 4.5]. Let $H<G$ be a finitely generated relatively quasiconvex subgroup and let $g \in G \backslash H$. There is a finite subset $F \subset G$ depending on $H$ and $g$ with the following property.

If $\pi: G \rightarrow G\left(N_{1}, \ldots, N_{m}\right)$ is an $H$-filling that is injective on $F$, then $\pi(H)$ is a relatively quasiconvex subgroup of $G\left(N_{1}, \ldots, N_{m}\right)$, and $\pi(g) \notin \pi(H)$.

Remark 4.5. Agol, Groves and Manning use in [2009, Propositions 4.3 and 4.5] a different definition of relative quasiconvexity than the one we use here. In particular, their definition requires the subgroup to be finitely generated. We show in Appendix A (specifically Corollary A.11) that their definition is equivalent to the one we use here under the assumption that the subgroup is finitely generated.

Proof of Theorem 1.9. Suppose every subgroup in $\mathscr{P}$ is LERF and slender. Let $Q$ be a relatively quasiconvex subgroup of $G$, and let $g$ be an element of $G$ not in $Q$. By Theorem 1.7, there is a fully quasiconvex subgroup $H$ that contains $Q$ and not $g$. We must choose filling kernels $\left\{N_{i}\right\}_{i=1}^{m}$ such that $\pi: G \rightarrow G\left(N_{1}, \ldots, N_{m}\right)$ is an $H$-filling, $G\left(N_{1}, \ldots, N_{m}\right)$ is a word hyperbolic group, $\pi(H)$ is a quasiconvex subgroup, and $\pi(g) \notin \pi(H)$.

By Theorem 3.6, there is a set $\left\{K_{1}, \ldots, K_{n}\right\}$ of representatives of the infinite maximal parabolic subgroups of $H$ up to conjugacy in $H$. For each $r \in\{1, \ldots, n\}$, there is an integer $i_{r} \in\{1, \ldots, m\}$ and an element $f_{r} \in G$ such that $K_{r}^{f_{r}}$ is a finite index subgroup of $P_{i_{r}}$. The index $i_{r}$ is determined by $r$, but there may be many distinct $f_{r}$ with this property. On the other hand, there will be only finitely many conjugates $K_{i}^{g}$ in $P_{i_{r}}$. Let $I_{r}$ be the intersection of these conjugates; the group $I_{r}$ is a finite index normal subgroup of $P_{i_{r}}$. For $i \in\{1, \ldots, m\}$ define the subgroup $M_{i}$ of $P_{i}$ as

$$
M_{i}= \begin{cases}P_{i} & \text { if }\left\{r \mid i_{r}=i\right\}=\varnothing, \\ \bigcap\left\{I_{r} \mid i_{r}=i\right\} & \text { if }\left\{r \mid i_{r}=i\right\} \neq \varnothing .\end{cases}
$$

Put another way, if some conjugate of $H$ intersects $P_{i}$ nontrivially,

$$
M_{i}=\bigcap\left\{K_{r}^{g} \mid K_{r}^{g} \cap P_{i} \neq\{1\}, r \in\{1, \ldots, n\} \text {, and } g \in G\right\} .
$$

In other words, $M_{i}$ is the intersection of $P_{i}$ with all the $K_{r}^{g}$ that lie in $P_{i}$. Because $M_{i}$ is a finite intersection of finite index normal subgroups of $P_{i}$, it is a finite index normal subgroup of $P_{i}$. From the definition, $M_{i}<K_{r}^{g}$ whenever $r \in\left\{r \mid i_{r}=i\right\}$ and $K_{r}^{g}<P_{i}$. 
By Theorem 4.4, there is a finite subset $F \subset G$ such that if $\pi: G \rightarrow \bar{G}$ is any $H$ filling that is injective on $F$, then $\pi(g) \notin \pi(H)$ and $\pi(H)$ is relatively quasiconvex. Let $B>0$ be the constant from Theorem 4.2, applied to this finite subset $F$.

Since each $P_{i}$ is residually finite, there is some finite index $\hat{N}_{i} \triangleleft P_{i}$ such that $|p|_{S}>B$ for all $p \in \hat{N}_{i} \backslash\{1\}$. Let $N_{i}=\hat{N}_{i} \cap M_{i}$. Since $N_{i}$ is an intersection of two finite index normal subgroups of $P_{i}$, it is a finite index normal subgroup of $P_{i}$. Moreover, $|p|_{S}>B$ for every $p \in N_{i} \backslash\{1\}$.

Lemma 4.6. $G \rightarrow G\left(N_{1}, \ldots, N_{m}\right)$ is an $H$-filling.

Proof. Let $P_{i} \in \mathscr{P}, f \in G$, and suppose that $H \cap P_{i}^{f} \neq\{1\}$. We must show that $N_{i}^{f}<H$. Obviously it suffices to show that $M_{i}^{f}<H$. Since $G$ is torsion free, $H \cap P_{i}^{f}$ is infinite and $H \cap P_{i}^{f}=K_{r}^{h}$ for some $h \in H$. The group

$$
M_{i}^{f}=\bigcap\left\{K_{r}^{g} \mid K_{r}^{g} \cap P_{i}^{f} \neq\{1\}, r \in\{1, \ldots, n\} \text {, and } g \in G\right\}
$$

must therefore be contained in $H \cap P_{i}^{f}$, and thus in $H$.

We claim the subgroup $H<G$ and the filling $G \rightarrow \bar{G}:=G\left(N_{1}, \ldots, N_{m}\right)$ satisfy the conclusions of Theorem 1.9. Indeed, (1) is satisfied by construction. By Theorem 4.2(2), the quotient $\bar{G}$ is hyperbolic relative to a set of finite groups; hence $\bar{G}$ is hyperbolic, which establishes (2). According to Lemma $4.6, G \rightarrow \bar{G}$ is an $H$-filling. By Theorem 4.2(3), $G \rightarrow \bar{G}$ is injective on $F$. Since the peripheral subgroups are slender, $H$ is finitely generated [Hruska 2008, Corollary 9.2]. We therefore may apply Theorem 4.4 to obtain finally parts (3) and (4).

\section{Applications to 3-manifolds}

5.1. Kleinian groups. A Kleinian group is a discrete subgroup of $\operatorname{Isom}\left(\mathbb{M}^{3}\right)$, the group of isometries of hyperbolic 3-space. We now will show that if all hyperbolic groups are residually finite, then all finitely generated Kleinian groups are LERF.

Proof of Corollary 1.5. By Selberg's lemma [Alperin 1987], every finitely generated Kleinian group contains a torsion-free subgroup of finite index. Applying Corollary 2.4, it suffices to consider torsion-free Kleinian groups. Let $G$ be a torsion-free Kleinian group and let $H<G$ be a finitely generated subgroup.

Case 1: $\mathbb{H}^{3} / G$ has finite volume. Suppose first that $H$ is not geometrically finite. By a result of [Canary 1996, Corollary 8.3] together with the positive solution to the tameness conjecture [Agol 2004; Calegari and Gabai 2006], $H$ must be a virtual fiber subgroup of $G$. This implies that there is a finite index subgroup of $G_{0}<G$ whose intersection $H_{0}$ with $H$ is normal in $G_{0}$, and such that $G_{0} / H_{0} \cong \mathbb{Z}$. The group $H_{0}$ is clearly separable in $G_{0}$, so $H$ is separable in $G$ by Corollary 2.4.

If $H$ is geometrically finite, then it is relatively quasiconvex by Theorem 1.3, and we may apply Theorem 1.2. 
Case 2: $\mathbb{M}^{3} / G$ has infinite volume. In this case it follows from the Scott core theorem and Thurston's geometrization theorem for Haken manifolds that $G$ is isomorphic to a geometrically finite Kleinian group $G^{\prime}$; see for example [Matsuzaki and Taniguchi 1998, Theorem 4.10]. If $H^{\prime}$ is the image of $H$ in $G^{\prime}$, then obviously $H$ is separable in $G$ if and only if $H^{\prime}$ is separable in $G^{\prime}$. We therefore may as well assume that $G$ is geometrically finite to begin with.

Since $G$ is geometrically finite and infinite covolume, every finitely generated subgroup of $G$ is geometrically finite by an argument of Thurston; for a proof, see [Morgan 1984, Proposition 7.1] or [Matsuzaki and Taniguchi 1998, Theorem 3.11]. In particular, $H$ is geometrically finite, so Theorem 1.3 implies that $H$ is a relatively quasiconvex subgroup of $G$. To finish, we apply Theorem 1.2 again.

\subsection{Subgroup separability in finite volume 3-manifolds.}

Proposition 5.3. If all fundamental groups of compact hyperbolic 3-manifolds are $Q C E R F$, then all fundamental groups of finite volume hyperbolic 3-orbifolds are $L E R F$.

Proof. Let $G=\pi_{1}(M)$, where $M$ is some finite volume hyperbolic 3-orbifold. Applying Corollary 2.4, we may pass to a finite cover and assume that $M$ is an orientable manifold. It follows that $G$ is hyperbolic relative to some finite collection $\mathscr{P}=\left\{P_{i}, \ldots, P_{m}\right\}$ of subgroups, each of which is isomorphic to $\mathbb{Z} \oplus \mathbb{Z}$.

Now suppose $Q<G$ is some finitely generated subgroup. We must show that $Q$ is separable. If $Q$ is geometrically infinite, then we argue as we did in the proof of Corollary 1.5 that $Q$ is the fundamental group of a virtual fiber, and thus separable. We may thus suppose that $Q$ is geometrically finite, and therefore a relatively quasiconvex subgroup of $G$.

Let $g \in G \backslash Q$. We then apply Theorem 1.7 to enlarge $Q$ to a fully quasiconvex subgroup $H$ not containing $g$. Let $F \subset G$ be the finite set obtained by applying Theorem 4.4 to $G, H$, and $g$. Let $B_{1}$ be the constant from Theorem 4.2 applied to $G$ and $F$ with respect to some generating set $S$ for $G$. We will choose a cyclic filling kernel $N_{i}<P_{i}$ for each $P_{i} \in \mathscr{P}$. The hyperbolic Dehn surgery theorem of Thurston [Thurston 1980; Hodgson and Kerckhoff 2005] implies there is some constant $B_{2}$ such that if the generators of the $N_{i}$ are chosen to have length greater that $B_{2}$, then the filling $G\left(N_{1}, \ldots, N_{m}\right)$ will be the (orbifold) fundamental group of a hyperbolic orbifold obtained by attaching orbifold solid tori to the boundary components of a compact core of $M$. Let $B=\max \left\{B_{1}, B_{2}\right\}$.

For each $P_{i} \in \mathscr{P}$, we choose some cyclic $N_{i}<P_{i}$. For each $i$, let $n_{i} \in P_{i}$ satisfy $\left|n_{i}\right|_{S}>B$. There are at most finitely many conjugates $P_{i}^{t_{1}}, \ldots P_{i}^{t_{k}}$ such that $P_{i}^{t_{j}} \cap H$ is nonempty; for each such $j$, the group $P_{i}^{t_{j}} \cap H$ is finite index in $P_{i}^{t_{j}}$. If there are no such conjugates, we choose $N_{i}=\left\langle n_{i}\right\rangle$. Otherwise, we let $N_{i}=\left\langle n_{i}^{\alpha}\right\rangle$, where the power $\alpha \in \mathbb{N}$ is chosen so that $n_{i}^{\alpha} \in H^{t_{j}{ }^{-1}}$ for each $j$, and so $\left|n_{i}^{\alpha}\right|_{S}>B$. 
With the $N_{i}<P_{i}$ chosen as above, the $H$-filling $G\left(N_{1}, \ldots, N_{m}\right)$ is a compact hyperbolic 3-orbifold group. Let $\pi: G \rightarrow G\left(N_{1}, \ldots, N_{m}\right)$ be the quotient map. By Theorem 4.4, $\pi(H)$ is a quasiconvex subgroup of $G\left(N_{1}, \ldots, N_{m}\right)$ that does not contain $\pi(g)$. By assumption, compact hyperbolic 3-manifold groups (and thus compact hyperbolic 3-orbifold groups) are QCERF. There is therefore some finite group $F$ and some $\phi: G\left(N_{1}, \ldots, N_{m}\right) \rightarrow F$ with $\phi(\pi(g)) \notin \phi(\pi(H))$. Since $\phi(\pi(H))$ contains $\phi(\pi(Q))$, we have separated $g$ from $Q$ in a finite quotient.

Remark 5.4. No part of the proof of Proposition 5.3 relies essentially on the results in this paper or those in [Agol et al. 2009]; the proof depends only on facts about hyperbolic 3-manifolds and 3-orbifolds that could be deduced by geometric arguments, based on the Gromov-Thurston $2 \pi$ theorem. On the other hand, the proposition illustrates nicely the general principle that a relatively hyperbolic group that can be approximated by QCERF Dehn fillings must itself be QCERF.

\section{Acknowledgments}

Thanks very much to Ian Agol and Daniel Groves, both for useful conversations and for helpful comments on an earlier draft of this note. We also thank the referee for insightful comments and corrections.

\section{Appendix A. The equivalence of definitions of quasiconvexity}

This paper relies heavily on [Martínez-Pedroza 2009] and [Agol et al. 2009] for properties of relatively quasiconvex subgroups of relatively hyperbolic groups. These papers use different definitions of relative quasiconvexity, but we show here that the two definitions agree, at least for finitely generated subgroups.

A few words about the literature. Dahmani [2003] and Osin [2006] studied classes of subgroups of relatively hyperbolic groups that they called relatively quasiconvex, a notion intended to generalize that of quasiconvexity in hyperbolic groups. Dahmani's definition was dynamical, whereas Osin's was Definition 3.4, also used in [Martínez-Pedroza 2009]. Hruska [2008] gave several definitions of relative quasiconvexity in the setting of countable (but not necessarily finitely generated) relatively hyperbolic groups, including definitions based on Osin's and Dahmani's, and showed that they are equivalent. The authors of [Agol et al. 2009] were mainly interested in relatively hyperbolic structures on groups that were already hyperbolic, and used a definition of relative quasiconvexity (based more closely on the usual metric notion of quasiconvexity) different from any of those in [Hruska 2008]. The definition in [Agol et al. 2009] applies only to a finitely generated subgroup of a finitely generated relatively hyperbolic group.

Throughout this section $G$ will be hyperbolic, relative to a finite collection of subgroups $\mathscr{P}=\left\{P_{1}, \ldots, P_{n}\right\}$, and $S$ will be a finite generating set for $G$. The 
cusped space (recalled below) for $G$ with respect to $\mathscr{P}$ and $S$ will be denoted by $X(G, \mathscr{P}, S)$, and $d(\cdot, \cdot)$ will denote the path metric on the cusped space. In particular, we will not need the word metric on $G$, but only the metric induced by this path metric. For detailed definitions and background on cusped spaces for relatively hyperbolic groups, see [Groves and Manning 2008] and [Hruska 2008]; we do sketch the construction and recall some terminology here.

Let $A$ be a discrete metric space with metric $\rho$. The combinatorial horoball based on $A$ is a graph $\mathscr{H}(A)$ with vertex set $A \times \mathbb{Z}_{\geq 0}$, such that

- $(a, n)$ is connected by an edge to $(a, n+1)$ for any $a \in A$, and

- for $n \geq 1,(a, n)$ is connected to $\left(a^{\prime}, n\right)$ whenever $\rho\left(a, a^{\prime}\right) \leq 2^{n}$.

Edges of the first type are called vertical; edges of the second type are called horizontal. We say that a vertex $(a, n)$ of $\mathscr{H}(A)$ has depth $n$. If $A$ is a subset of a path metric space $Y$, we may attach a horoball to $Y$ along $A$ by gluing $A \subseteq Y$ to $A \times\{0\} \subset \mathcal{H}(A)$, and taking the obvious path metric on the union. If $G$ is finitely generated by $S$, we take $Y$ to be the Cayley graph of $G$ with respect to $S$; any subset of $G$ inherits a discrete metric from the path metric on the Cayley graph. The cusped space $X(G, \mathscr{P}, S)$ is the space obtained by simultaneously attaching horoballs to $Y$ along all left cosets $t P$ for $P \in \mathscr{P}$. A vertex $v$ of $X(G, \mathscr{P}, S)$ corresponds either to a group element, if it lies in the Cayley graph of $G$, or otherwise to a triple $(t P, g, n)$, where $t P$ is a left coset of an element of $\mathscr{P}$, the element $g$ lies in $t P$, and $n>0$ is the depth of $v$ in the attached horoball $\mathscr{H}(t P)$. In what follows we do not distinguish between $v$ and the corresponding group element or triple.

The group $G$ is hyperbolic relative to $\mathscr{P}$ if and only if the space $X(G, \mathscr{P}, S)$ is Gromov hyperbolic; see for example [Groves and Manning 2008, Theorem 3.25]. If so, then $G$ acts on $X(G, \mathscr{P}, S)$ geometrically finitely, ${ }^{1}$ meaning in particular this:

(1) Given any $n \geq 0$, let $X_{n}$ be the subset of $X(G, \mathscr{P}, S)$ obtained by deleting all vertices of height greater than $n$. Then $G$ acts cocompactly on $X_{n}$, which is an example of what Hruska calls a truncated space for the action of $G$ on $X(G, \mathscr{P}, S)$.

(2) For fixed $n$, there are only finitely many components of $X(G, \mathscr{P}, S) \backslash X_{n}$, up to the action of $G$. (The components of $X(G, \mathscr{P}, S) \backslash X_{n-1}$ are called $n$-horoballs for $n \geq 1$. If $n$ is understood, we call them horoballs. A 0 -horoball is a 1neighborhood of a 1-horoball, and is equal to $\mathscr{H}(t P)$ for some coset $t P$ of some $P \in \mathscr{P}$.)

Relatively quasiconvex subgroups according to Agol, Groves, and Manning. Let $H$ be a relatively hyperbolic group, let $\mathscr{D}=\left\{D_{1}, \ldots, D_{m}\right\}$ be the peripheral subgroups of $H$, and let $T$ be a finite generating set for $H$. Let $\phi: H \rightarrow G$ be a

\footnotetext{
${ }^{1}$ Hruska uses the term "cofinitely".
} 
homomorphism. If every $\phi\left(D_{i}\right) \in \mathscr{D}$ is conjugate in $G$ into some $P_{j} \in \mathscr{P}$, we say that the map $\phi$ respects the peripheral structure on $H$.

One can extend such a map $\phi$ to a map $\check{\phi}$ between zero-skeletons of cusped spaces as follows: For each $D_{i} \in \mathscr{D}$, choose an element $c_{i} \in G$ (of minimal length) and some $P_{j_{i}}$ such that $\phi\left(D_{i}\right) \subseteq c P_{j_{i}} c^{-1}$. For $h \in H$, we have $\check{\phi}(h)=\phi(h)$. For a vertex $\left(s D_{i}, h, n\right)$ in a horoball of $X(H, \mathscr{D}, T)$, define

$$
\check{\phi}\left(s D_{i}, h, n\right)=\left(\phi(s) c_{i} P_{j_{i}}, \phi(h) c_{i}, n\right) .
$$

Lemma A.1 [Agol et al. 2009, Lemma 3.1]. Let $\phi: H \rightarrow G$ be a homomorphism that respects the peripheral structure on $H$. The extension $\check{\phi}$ defined above is $H$-equivariant and Lipschitz. If $\phi$ is injective, then $\breve{\phi}$ is proper.

Recall that for $C \geq 0$ a subset $A$ of a geodesic metric space $X$ is $C$-quasiconvex if every geodesic with endpoints in $A$ lies in a $C$-neighborhood of $A$, and is quasiconvex if it is $C$-quasiconvex for some $C$. The following is slightly paraphrased:

Definition A.2 [Agol et al. 2009, Definition 3.11]. Let $G$ be as above, and let $H<G$ be finitely generated by a set $T$. We say that $H$ is $(Q C-A G M)$ relatively quasiconvex in $(G, \mathscr{P})$ if, for some finite collection of subgroups $\mathscr{D}$ of $H$,

(1) $H$ is hyperbolic relative to $\mathscr{D}$, and

(2) if $\imath: H \rightarrow G$ is the inclusion, then the map $\check{\imath}: X(H, \mathscr{D}, T)^{0} \rightarrow X(G, \mathscr{P}, S)^{0}$ described in Lemma A.1 has quasiconvex image.

Relatively quasiconvex subgroups according to Hruska. The following definition is [Hruska 2008, Definition 6.6], where it is called $Q C$-3. Hruska shows there that this definition is equivalent to several others, including our Definition 3.4.

Definition A.3. We say a subgroup $H \leq G$ is $(Q C-H)$ relatively quasiconvex if the following holds. Let $(X, \rho)$ be some (any) proper Gromov hyperbolic space on which $(G, \mathscr{P})$ acts geometrically finitely. Let $X-U$ be some (any) truncated space for $G$ acting on $X$. For some (any) basepoint $x \in X-U$, there is a constant $\mu \geq 0$ such that whenever $c$ is a geodesic in $X$ with endpoints in the orbit $H x$, we have $c \cap(X-U) \subseteq \mathcal{N}_{\mu}(H x)$, where the neighborhood is taken with respect to the metric $\rho$ on $X$.

Remark A.4. The meaning of "some (any)" in Definition A.3 just means that the word "some" can be replaced by "any" without affecting which subgroups of $G$ are $(\mathrm{QC}-\mathrm{H})$ relatively quasiconvex. Thus this definition has some nondefinitional content, established in [Hruska 2008, Proposition 7.5 and 7.6].

Definition A.5. Let $A \subset X=X(G, \mathscr{P}, S)$ be a horoball, and let $R>0$. A geodesic $\gamma$ penetrates the horoball $A$ to depth $R$ if there is a point $p \in \gamma \cap A$ at distance at least $R$ from $X \backslash A$. We say that $A$ is $R$-penetrated by the subgroup $H$ if there is a geodesic $\gamma$ with endpoints in $H$ that penetrates the horoball $A$ to depth $R$. 
Proposition A.6. Let $H<G$ be $(Q C-H)$ relatively quasiconvex. Then there is a constant $R=R(G, \mathscr{P}, S, H)$ such that whenever a 0 -horoball is $R$-penetrated by $H$, the intersection of $H$ with the stabilizer of that horoball is infinite.

Before the proof, we quote a proposition from [Hruska 2008] and prove two lemmas.

Proposition A.7 [Hruska 2008, Proposition 9.4]. Suppose G has a proper, left invariant metric $d$, and suppose $x H$ and $y K$ are arbitrary left cosets of subgroups of $G$. For each constant $L$, there is a constant $L^{\prime}=L^{\prime}(G, d, x H, y K)$ such that in the metric space $(G, d)$, we have

$$
\mathcal{N}_{L}(x H) \cap \mathcal{N}_{L}(y K) \subseteq \mathcal{N}_{L^{\prime}}\left(x H x^{-1} \cap y K y^{-1}\right) .
$$

Lemma A.8. Let $H$ be a $(Q C-H)$ relatively quasiconvex subgroup of $G$. Let $A$ be a 0 -horoball of $X(G, \mathscr{P}, S)$ whose stabilizer is $P^{t}$ for $P \in \mathscr{P}$. If $A$ is $R$-penetrated by $H$ for all $R>0$, then $H \cap P^{t}$ is infinite.

Proof. It suffices to show that, for every $M>0$, there is some $h$ in $H \cap P^{t}$ with $d(1, h)>M$.

Let $\mu$ be the quasiconvexity constant of Definition A.3 for $H$ and the space $X^{\prime}$ that consists of all vertices in $X(G, \mathscr{P}, S)$ at depth 0 . Let $C$ be the constant given by Proposition A.7 such that $\mathcal{N}_{\mu}(H) \cap t P \subseteq \mathcal{N}_{C}\left(H \cap t P t^{-1}\right)$, where the neighborhoods are taken in the cusped space.

Suppose that $\gamma$ is a geodesic with endpoints in $H$ that penetrates the horoball $A$ to depth $M+C$. The first and last points of $\gamma \cap A$ are group elements $a$ and $b$, both in the coset $t P$. Since $H$ is (QC-H) relatively quasiconvex, $a$ and $b$ are elements of $\mathcal{N}_{\mu}(H) \cap t P$ and therefore (using Proposition A.7) there are elements $h_{1}$ and $h_{2}$ in $H \cap P^{t}$ such that $d\left(h_{1}, a\right) \leq C$ and $d\left(h_{2}, b\right) \leq C$. Since $d(a, b) \geq 2(M+C)$,

$$
d\left(1, h_{1}^{-1} h_{2}\right)=d\left(h_{1}, h_{2}\right) \geq 2(M+C)-2 C \geq 2 M>M .
$$

Lemma A.9. Let $H$ be a $(Q C-H)$ relatively quasiconvex subgroup of $G$. Let $\mu$ be the quasiconvexity constant of Definition A.3 for $H$ and the space $X^{\prime}=X_{0}$ that is obtained from $X(G, \mathscr{P}, S)$ by deleting all vertices of positive depth.

Let $R>0$, and let $A$ be a 0 -horoball stabilized by $P^{t}$ for $P \in \mathscr{P}$. If $A$ is $R$ penetrated by $H$, then there is a horoball $A^{\prime}$ such that

(1) $A^{\prime}=h A$ for some $h \in H$,

(2) $d\left(A^{\prime}, 1\right) \leq \mu$, and

(3) $A^{\prime}$ is R-penetrated by $H$.

Proof. Suppose that $\gamma$ is a geodesic with endpoints $h_{1}$ and $h_{2}$ in $H$ that penetrates the horoball $A$ to depth $R$. Let $a$ and $b$ be the first and last vertices of $\gamma \cap A$. By (QC-H) relative quasiconvexity, there is some $h \in H$ such that $d(a, h) \leq \mu$. 
The geodesic $h^{-1} \gamma$ goes between $h^{-1} h_{2}$ and $h^{-1} h_{2}$, and penetrates the horoball $A^{\prime}=h^{-1} A$ to depth $R$. Moreover, $d\left(1, A^{\prime}\right) \leq d\left(1, h^{-1} a\right)=d(a, h) \leq \mu$.

Proof of Proposition A.6. Suppose there is no such number $R$. There must be a sequence of integers $R_{i} \rightarrow \infty$ and a sequence of 0-horoballs $\left\{A_{i}\right\}$ such that, for each $i$, the horoball $A_{i}$ is $R_{i}$-penetrated by $H$ but the intersection of the stabilizer of $A_{i}$ with $H$ is finite.

For $h \in H$, the stabilizer of $h A_{i}$ is conjugate (by $h$ ) to the stabilizer of $A_{i}$. Using Lemma A.9, we can therefore assume that $d\left(1, A_{i}\right) \leq \mu$ for each $i$. By passing to a subsequence, we can therefore assume that the sequence $\left\{A_{i}\right\}$ is constant. It follows that $A_{0}$ is $R_{i}$-penetrated by $H$ for all $i$. Lemma A.8 then implies that the intersection of $H$ with the stabilizer of $A_{0}$ is infinite, which is a contradiction.

Equivalence of the two definitions. Suppose $G$ is a group hyperbolic relative to a finite collection of subgroups $\mathscr{P}$, and let $S$ be a finite generating set for G. Let $X(G, \mathscr{P}, S)$ be the cusped space for $G$ with respect to $\mathscr{P}$ and $S$, and let $\delta$ be its hyperbolicity constant.

Theorem A.10. Let $H$ be a finitely generated subgroup of $G$. Then $H$ is $(Q C-H)$ relatively quasiconvex if and only if $H$ is $(Q C-A G M)$ relatively quasiconvex.

Proof. One direction is easy. Suppose that $H<G$ is (QC-AGM) relatively quasiconvex, is generated by the finite set $T$, and has peripheral subgroups $\mathscr{D}$. Recall that to define $\check{\imath}: X(H, \mathscr{D}, T)^{0} \rightarrow X(G, \mathscr{P}, S)^{0}$, an element $c_{i} \in G$ was chosen for each $D_{i} \in \mathscr{D}$ so that $D_{i} \subset c_{i} P_{j_{i}} c_{i}^{-1}$. Let $C=\max \left\{d\left(1, c_{i}\right) \mid D_{i} \in \mathscr{D}\right\}$, and let $C_{q}$ be the constant of quasiconvexity in the definition of (QC-AGM) quasiconvexity. As remarked at the beginning of the appendix, the cusped space $X=X(G, \mathscr{P}, S)$ is acted on geometrically finitely by $G$, and the subspace $X-U=X_{0} \subset X(G, \mathscr{P}, S)$ obtained by deleting 1-horoballs is a truncated space for the action. Moreover, as explained in Remark A.4, it suffices to find a $\mu$ that works for this choice of $X$ and $X-U$ and for the $H$-orbit of 1 in $X$. Let $x, y \in H$, and let $\gamma$ be any geodesic joining them in $X$. Let $z$ be a vertex of $\gamma$ contained in $X_{0}$. By (QC-AGM), there is some point $w \in \check{l}\left(X(H, \mathscr{D}, T)^{0}\right)$ such that $d(z, w) \leq C_{q}$. It follows that $w$ belongs to $X_{C_{q}}$, but any point in $\breve{\imath}(H, \mathscr{D}, T) \cap X_{C_{q}}$ is at most $C+C_{q}$ away from some point in $H$. It follows that $z$ is no further than $\mu:=C+2 C_{q}$ from $H$, and so $H$ is (QC-H) relatively quasiconvex.

For the other direction, let $H$ be a subgroup of $G$, and suppose that $H$ is (QC-H) relatively quasiconvex. Let $\mathscr{D}$ be a set of representatives of the $H$-conjugacy classes of infinite maximal parabolic subgroups of $H$. By [Hruska 2008, Theorem 9.1], $H$ is hyperbolic relative to $\mathscr{D}$. By Lemma A.1, the inclusion $\imath: H \rightarrow G$ extends to a Lipschitz map of (0-skeletons of) cusped spaces $\check{\imath}: X(H, \mathscr{D}, T)^{0} \rightarrow X(G, \mathscr{P}, S)^{0}$. We need to prove that the image $Y=\breve{l}\left(X(H, \mathscr{D}, T)^{0}\right)$ of $\breve{l}$ is quasiconvex. 
Let $R$ be the constant provided by Proposition A.6 for the subgroup $H$. Let $X^{\prime}=X_{100 \delta+R}$ be the subspace of $X(G, \mathscr{P}, S)$ consisting of all vertices at depth at most $100 \delta+R$. Since $H$ is $(\mathrm{QC}-\mathrm{H})$ relatively quasiconvex, there is a constant $\mu$ such that $\zeta \cap X^{\prime} \subset \mathcal{N}_{\mu}(H) \subset \mathcal{N}_{\mu}(Y)$ for any geodesic $\zeta$ in $X(G, \mathscr{P}, S)$ with endpoints in $H$, where the neighborhoods are taken with respect to the metric on $X(G, \mathscr{P}, S)$.

Let $x$ and $y$ be vertices of $Y$ and let $\gamma$ be a geodesic between them. We will show that the vertices of $\gamma$ are contained in the $M$-neighborhood of $Y$, where $M$ is a constant independent of $x, y$, and $\gamma$. We divide the proof into five (not necessarily disjoint) cases.

Case 1: The points $x$ and $y$ lie deeper than $10 \delta$ in the same horoball. By recalling some easy properties of the geometry of horoballs, we will show that $\gamma$ is contained in the $M_{1}$-neighborhood of $Y$, where $M_{1}=6$. First, the $10 \delta$-horoball containing $x$ and $y$ is convex; see [Groves and Manning 2008, Lemma 3.26]. Second, any geodesic with the same endpoints as $\gamma$ is Hausdorff distance at most 4 from $\gamma$. Finally, there is a geodesic $\gamma^{\prime}$ of a particularly nice form with the same endpoints as $\gamma$. The geodesic $\gamma^{\prime}$ is a regular geodesic, which means that all its edges are vertical except for at most three consecutive horizontal edges at maximum depth; see [Groves and Manning 2008, Lemma 3.10]. Since the vertical subsegments of $\gamma^{\prime}$ start at points in $Y$ and are vertical, they stay in $Y$, and so $\gamma^{\prime}$ stays in a 2-neighborhood of $Y$. As $\gamma$ is contained in a 4-neighborhood of $\gamma^{\prime}$, we have $\gamma$ contained in a 6-neighborhood of $Y$.

Case 2: The points $x$ and $y$ are elements of $H$, they are in the neighborhood of radius $\mu$ of a horoball $\mathscr{H}(t P)$, and the geodesic $\gamma$ penetrates the horoball $\mathscr{H}(t P)$ to depth larger than $100 \delta+R$. In this case, we will approximate $\gamma$ by a regular geodesic inside $\mathcal{H}(t P)$ with (possibly different) endpoints in $Y$. Without loss of generality, assume that $x$ is the identity, and so $d(1, t) \leq \mu$.

By Proposition A.6, the intersection $H \cap P^{t}$ is infinite, from which it follows that $H \cap P^{t}=D^{s}$ for some $D \in \mathscr{D}$ and $s \in H$. We claim $s$ can be chosen so that $d(1, s)<K$ for a constant $K$ independent of $x, y$, and $\gamma$. Indeed, we observe that the set

$$
W=\left\{(r, P) \in G \times \mathscr{P} \mid d(1, r) \leq \mu, \#\left(H \cap P^{r}\right)=\infty\right\}
$$

is finite. For each $w=(r, P) \in W$, choose $u_{w} \in H$ so that $H \cap P^{r}=D^{u}$ for some $D \in \mathscr{D}$; we let $K$ be the maximum of $d\left(1, u_{w}\right)$ over all $w \in W$.

We further claim that there is an element $y^{\prime} \in H \cap P^{t}$ such that $d\left(y, y^{\prime}\right) \leq L$ for a constant $L$ independent of $x, y$, and $\gamma$. Indeed, for each $w=(r, P)$ in the set $W$ defined above, Proposition A.7 implies we can find an $L_{w}>0$ such that

$$
H \cap \mathcal{N}_{\mu}(r P) \subseteq \mathcal{N}_{L_{w}}\left(H \cap P^{r}\right) ;
$$

we let $L$ be the maximum $L_{w}$ over all $w \in W$. 
Recall that to define $\check{\imath}: X(H, \mathscr{D}, T)^{0} \rightarrow X(G, \mathscr{P}, S)^{0}$, an element $c_{i} \in G$ was chosen for each $D_{i} \in \mathscr{D}$ so that $D_{i} \subset c_{i} P_{j_{i}} c_{i}^{-1}$. Let

$$
C=\max \left\{d\left(1, c_{i}\right) \mid D_{i} \in \mathscr{D}\right\}
$$

The subgroup $D$ is equal to $D_{i}$ for some $i$, and we set $c=c_{i}$ for the same $i$.

Consider the elements $(s D, s, 10 \delta)$ and $\left(s D, y^{\prime} s, 10 \delta\right)$ of $X(H, \mathscr{D}, T)$ and their corresponding images in $Y$ given by $(s c P, s c, 10 \delta)$ and $\left(s c P, y^{\prime} s c, 10 \delta\right)$. The points $(s c P, s c, 10 \delta)$ and $\left(s c P, y^{\prime} s c, 10 \delta\right)$ belong to the same $10 \delta$-horoball, which is convex in $X(G, \mathscr{P}, S)$, as we noted in Case 1. Also as noted in Case 1, there is a regular geodesic $\gamma^{\prime}$ joining the points $(s c P, s c, 10 \delta)$ and $\left(s c P, y^{\prime} s c, 10 \delta\right)$; since the endpoints lie in $Y$, the geodesic $\gamma^{\prime}$ is contained in the 2-neighborhood of $Y$.

On the other hand, the endpoints of the geodesics $\gamma$ and $\gamma^{\prime}$ are close, namely,

$$
\begin{aligned}
d(1,(s c P, s c, 10 \delta)) & \leq d(1, s)+d(1, c)+10 \delta \leq K+C+10 \delta \text { and } \\
d\left(y,\left(s c P, y^{\prime} s c, 10 \delta\right)\right) & \leq d\left(y, y^{\prime}\right)+d\left(y^{\prime},\left(s c P, y^{\prime} s c, 10 \delta\right)\right) \leq L+K+C+10 \delta .
\end{aligned}
$$

Since $X(G, \mathscr{P}, S)$ is $\delta$-hyperbolic, the Hausdorff distance between $\gamma^{\prime}$ and $\gamma$ is at most the distance between endpoints plus $2 \delta$. Thus if $M_{2}=2 \delta+K+L+C+10 \delta$, then $\gamma$ is contained in the $M_{2}$-neighborhood of $Y$. This completes this case.

Case 3: $x$ and $y$ are elements of $H$. We split $\gamma$ into subsegments $\gamma_{1}, \gamma_{2}, \ldots, \gamma_{k}$ such that no $\gamma_{i}$ contains any group element (depth 0 vertex) in its interior, but the endpoints of each $\gamma_{i}$ are group elements. Observe that each $\gamma_{i}$ is either a single edge or a geodesic segment contained in a 0-horoball. Furthermore, since $H$ is (QC-H) relatively quasiconvex, the endpoints of each $\gamma_{i}$ are contained in the $\mu$-neighborhood of $H$. We claim that each $\gamma_{i}$ is contained in the $M_{3}$-neighborhood of $Y$, where $M_{3}=110 \delta+R+3 \mu+2+M_{2}$. If $\gamma_{i}$ is an edge, then the claim is immediate, so we suppose $\gamma_{i}$ is contained in a 0 -horoball $\mathscr{A}$.

First, suppose $\gamma_{i}$ does not penetrate $\mathscr{A}$ to depth $110 \delta+R+2 \mu$. An easy argument shows that the length of a geodesic in a combinatorial horoball is at most twice its maximum depth plus 4 , so we have $\left|\gamma_{i}\right|<220 \delta+2 R+2 \mu+4$, and $\gamma_{i}$ is therefore contained in the $(110 \delta+R+3 \mu+2)$-neighborhood of $H$. In particular, $\gamma_{i}$ is contained in the $M_{3}$-neighborhood of $Y$.

Now suppose that $\gamma_{i}$ does penetrate the horoball $\mathscr{A}$ to depth $110 \delta+R+2 \mu$. Let $h_{1}$ and $h_{2}$ be elements of $H$ that are at distance at most $\mu$ from the endpoints of $\gamma_{i}$, and let $\alpha$ be a geodesic between them. Since $X(G, \mathscr{P}, S)$ is $\delta$-hyperbolic, the Hausdorff distance between $\gamma_{i}$ and $\alpha$ is at most $2 \delta+\mu$. It follows that $\alpha$ penetrates the horoball $\mathscr{A}$ to depth $100 \delta+R+\mu$, and hence it satisfies the condition of Case 2. Therefore, $\gamma_{i}$ is in the $\left(2 \delta+\mu+M_{2}\right)$-neighborhood (and hence in the $M_{3}$-neighborhood) of $Y$. 
Case 4: $x$ and $y$ lie at depth no more than $50 \delta$ in $X(G, \mathscr{P}, S)$. If $x \in Y$ lies in a 1-horoball, then $x=\left(t P, h c_{i}, n\right)$ for some $P \in \mathscr{P}$, some $h \in H$, some $i \in\{1, \ldots, m\}$, and some $n \leq 50 \delta$; otherwise, $x \in H$. In any case, there is an element $h_{1} \in H$ such that $d\left(x, h_{1}\right) \leq 50 \delta+C$, where $C$ is the constant defined in (A-1). By the same argument, there is an element $h_{2} \in H$ such that $d\left(x, h_{2}\right) \leq 50 \delta+C$. Since $X(G, \mathscr{P}, S)$ is $\delta$-hyperbolic, the Hausdorff distance between $\gamma$ and any geodesic $\gamma^{\prime}$ between $h_{1}$ and $h_{2}$ is at most $52 \delta+C$. We may apply Case 3 to $\gamma^{\prime}$, and deduce that $\gamma$ is contained in the $M_{4}$-neighborhood of $Y$, where $M_{4}=52 \delta+C+M_{3}$.

Case 5: Either $x$ or $y$ lies inside a $50 \delta$-horoball, but we are not in Case 1 . Here we follow the proof of the last case of [Agol et al. 2009, Proposition 3.12]. If $x$ or $y$ lies in a horoball, that point is connected by a vertical path to a point in the right coset $H c_{i}$ at depth 0 in $X(G, \mathscr{P}, S)$. It's therefore possible to modify $\gamma$ (by appending and deleting (mostly) vertical paths lying in a 3-neighborhood of $Y$ ) to a $10 \delta$-local geodesic $\gamma^{\prime}$ with endpoints within $C$ of $H$; the geodesic $\gamma$ is contained in a 3-neighborhood of $\gamma^{\prime} \cup Y$. By [Bridson and Haefliger 1999, III.H.1.13(3)], $\gamma^{\prime}$ is a $(7 / 3,2)$-quasigeodesic. Since quasigeodesics track geodesics, there is a constant $L_{Q}$ depending only on $\delta$ and $C$, and a geodesic $\gamma^{\prime \prime}$ with endpoints in $H$ such that the Hausdorff distance between $\gamma^{\prime}$ and $\gamma^{\prime \prime}$ is at most $L_{Q}$. By Case 3, $\gamma^{\prime \prime}$ is contained in the $M_{2}$-neighborhood of $Y$. Let $M_{5}=3+L_{Q}+M_{2}$, and observe that $\gamma$ is contained in the $M_{5}$-neighborhood of $Y$.

Finally, we set $M=\max \left\{M_{1}, \ldots, M_{5}\right\}$, and note that $M$ does not depend on the vertices $x$ and $y$ of $Y$, or on the geodesic $\gamma$ joining them. It follows that $Y=\breve{l}\left(X(H, \mathscr{D}, T)^{0}\right)$ is $M$-quasiconvex in $X(H, \mathscr{D}, T)$, and so $H$ is (QC-AGM) relatively quasiconvex in $(G, \mathscr{P})$.

By applying the main result of Hruska [2008] on the equivalence of various definitions of relative quasiconvexity - our Definition 3.4 is Hruska's (QC-5), and our Definition A.3 (QC-H) is Hruska's (QC-3) - we obtain a useful fact:

Corollary A.11. Suppose $G$ is hyperbolic relative to $\mathscr{P}$, and let $H$ be a finitely generated subgroup of $G$. The following are equivalent:

(1) $H$ is a relatively quasiconvex subgroup of $G$, in the sense of Definition 3.4.

(2) $H$ is a relatively quasiconvex subgroup of $G$, in the sense of Definition A.2.

\section{Appendix B. Extending the main result in the presence of torsion}

Here we give some idea of the changes necessary to prove Theorem 1.9 (and hence Theorem 1.2) in the presence of torsion. Let $G$ be a group hyperbolic relative to a finite collection $\mathscr{P}$ of LERF and slender subgroups, and let $H$ be some relatively quasiconvex subgroup of $G$. 
The main difference is that we must deal with the possibility that our relatively quasiconvex subgroup has finite but nontrivial maximal parabolic subgroups. Since a finite subgroup of a relatively hyperbolic group may intersect arbitrary sets of parabolic subgroups, we have to ignore these intersections. This is already handled in the arguments of Section 3 by only amalgamating with parabolic subgroups that have infinite intersection with $H$ to obtain the fully quasiconvex subgroup $Q$.

In Section 4, it is necessary to modify the definition of $H$-filling as follows:

Definition B.1 (alternative definition in the presence of torsion). Suppose $H$ is a relatively quasiconvex subgroup of $G$. A filling $G \rightarrow G\left(N_{1}, \ldots, N_{m}\right)$ is an $H$ filling if $N_{i}^{g} \subset P_{i}^{g} \cap H$ whenever $H \cap P_{i}^{g}$ is infinite.

We must check that the results from [Agol et al. 2009, Section 4.2] still hold with the new definition. (We do not know how to prove the result about height from Section 4.3 in this more general setting, but we do not need it for our argument.)

Examining the proofs in [Agol et al. 2009], the reader may check that it suffices to extend the technical Lemma 4.2 therein. We sketch how to do so, for the experts: In that lemma, the hypothesis of an $H$-filling is used to deduce the existence of a nontrivial element of $H$ that is also in a conjugate of a filling kernel fixing a certain horoball; this follows from the fact that a geodesic between elements of $H$ penetrates that horoball deeply. The heart of the argument is showing that if the geodesic penetrates the horoball deeply, the intersection of $H$ with the horoball stabilizer is infinite. In the torsion-free setting, it suffices to show that the intersection is nontrivial. The proof in the presence of torsion is given in the previous appendix as Proposition A.6. With this proposition, one can prove the extended version of Lemma 4.2 in a straightforward manner, choosing slightly different constants to take the constant $R$ from Proposition A.6 into account.

The proofs of [Agol et al. 2009, Propositions 4.3 and 4.5] go through in exactly the same way, and we obtain the same statement as Theorem 4.4 above, but with the new meaning of $H$-filling. Using Osin's Dehn filling result in place of Theorem 4.2, the rest of the proof of Theorem 1.9 goes through as written, with the exception that each mention of a condition of the form " $A \cap B \neq\{1\}$ " for $A$ and $B$ subgroups of $G$ should be replaced by " $A \cap B$ is infinite".

\section{References}

[Agol 2004] I. Agol, “Tameness of hyperbolic 3-manifolds”, preprint, 2004. arXiv math/0405568 [Agol et al. 2001] I. Agol, D. D. Long, and A. W. Reid, "The Bianchi groups are separable on geometrically finite subgroups", Ann. of Math. (2) 153:3 (2001), 599-621. MR 2002e:20099 Zbl 1067.20067

[Agol et al. 2009] I. Agol, D. Groves, and J. F. Manning, "Residual finiteness, QCERF and fillings of hyperbolic groups”, Geom. Topol. 13:2 (2009), 1043-1073. MR 2470970 Zbl 05530199 
[Alperin 1987] R. C. Alperin, “An elementary account of Selberg's lemma”, Enseign. Math. (2) 33:3-4 (1987), 269-273. MR 89f:20051 Zbl 0639.20030

[Bowditch 1995] B. H. Bowditch, "Geometrical finiteness with variable negative curvature", Duke Math. J. 77:1 (1995), 229-274. MR 96b:53056 Zbl 0877.57018

[Bowditch 1999] B. H. Bowditch, "Relatively hyperbolic groups", preprint, 1999, Available at http:// www.warwick.ac.uk/ masgak/papers/bhb-relhyp.pdf.

[Bridson and Haefliger 1999] M. R. Bridson and A. Haefliger, Metric spaces of non-positive curvature, Grundlehren der Mathematischen Wissenschaften 319, Springer, Berlin, 1999. MR 2000k: 53038 Zbl 0988.53001

[Calegari and Gabai 2006] D. Calegari and D. Gabai, "Shrinkwrapping and the taming of hyperbolic 3-manifolds”, J. Amer. Math. Soc. 19:2 (2006), 385-446. MR 2006g:57030 Zbl 1090.57010

[Canary 1996] R. D. Canary, "A covering theorem for hyperbolic 3-manifolds and its applications", Topology 35:3 (1996), 751-778. MR 97e:57012 Zbl 0863.57010

[Cooper and Long 1999] D. Cooper and D. D. Long, "Virtually Haken Dehn-filling", J. Differential Geom. 52:1 (1999), 173-187. MR 2001a:57025 Zbl 1025.57020

[Cooper and Long 2001] D. Cooper and D. D. Long, "Some surface subgroups survive surgery", Geom. Topol. 5 (2001), 347-367. MR 2002g:57031 Zbl 1009.57017

[Dahmani 2003] F. Dahmani, "Combination of convergence groups", Geom. Topol. 7 (2003), 933963. MR 2005g:20063 Zbl 1037.20042

[Farb 1998] B. Farb, "Relatively hyperbolic groups", Geom. Funct. Anal. 8:5 (1998), 810-840. MR 99j:20043 Zbl 0985.20027

[Groves and Manning 2008] D. Groves and J. F. Manning, "Dehn filling in relatively hyperbolic groups”, Israel J. Math. 168 (2008), 317-429. MR 2009h:57030 Zbl 05508729

[Hall 1949] M. Hall, Jr., "Coset representations in free groups”, Trans. Amer. Math. Soc. 67 (1949), 421-432. MR 11,322e Zbl 0035.01301

[Hirsch 1938] K. A. Hirsch, “On infinite soluble groups, I”, Proc. London Math. Soc. (2) 44 (1938), 53-60.

[Hodgson and Kerckhoff 2005] C. D. Hodgson and S. P. Kerckhoff, "Universal bounds for hyperbolic Dehn surgery”, Ann. of Math. (2) 162:1 (2005), 367-421. MR 2006g:57031 Zbl 1087.57011

[Hruska 2008] G. C. Hruska, "Relative hyperbolicity and relative quasiconvexity for countable groups", preprint, 2008. arXiv 0801.4596v2

[Kapovich and Wise 2000] I. Kapovich and D. T. Wise, "The equivalence of some residual properties of word-hyperbolic groups", J. Algebra 223:2 (2000), 562-583. MR 2001f:20086 Zbl 0951.20029

[Long and Niblo 1991] D. D. Long and G. A. Niblo, "Subgroup separability and 3-manifold groups", Math. Z. 207:2 (1991), 209-215. MR 92g:20047 Zbl 0711.57002

[Mal'cev 1958] A. I. Mal'cev, “On homomorphisms onto finite groups”, Učen. Zap. Ivanov. Gos. Ped. Inst. 18 (1958), 49-60. In Russian; translated in American Mathematical Society Translations (2) 119 (1983), 67-79.

[Martínez-Pedroza 2009] E. Martínez-Pedroza, "Combination of quasiconvex subgroups of relatively hyperbolic groups”, Groups Geom. Dyn. 3:2 (2009), 317-342. MR 2486802 Zbl 05550907

[Matsuzaki and Taniguchi 1998] K. Matsuzaki and M. Taniguchi, Hyperbolic manifolds and Kleinian groups, Oxford University Press, New York, 1998. MR 99g:30055 Zbl 0892.30035

[Minasyan 2006] A. Minasyan, "Separable subsets of GFERF negatively curved groups", J. Algebra 304:2 (2006), 1090-1100. MR 2007j:20064 Zbl 05077834 
[Morgan 1984] J. W. Morgan, “On Thurston's uniformization theorem for three-dimensional manifolds", pp. 37-125 in The Smith conjecture (New York, 1979), edited by J. W. Morgan and H. Bass, Pure Appl. Math. 112, Academic, Orlando, FL, 1984. MR 758464 Zbl 0599.57002

[Osin 2006] D. V. Osin, Relatively hyperbolic groups: Intrinsic geometry, algebraic properties, and algorithmic problems, Mem. Amer. Math. Soc. 179:843, Amer. Math. Soc., Providence, RI, 2006. MR 2006i:20047 Zbl 1093.20025

[Osin 2007] D. V. Osin, "Peripheral fillings of relatively hyperbolic groups", Invent. Math. 167:2 (2007), 295-326. MR 2008d:20080 Zbl 1116.20031

[Thurston 1980] W. P. Thurston, "Geometry and topology of three-manifolds", lecture notes, Princeton University, 1980, Available at http://www.msri.org/publications/books/gt3m/.

[Wise 2002] D. T. Wise, "The residual finiteness of negatively curved polygons of finite groups", Invent. Math. 149:3 (2002), 579-617. MR 2003e:20033 Zbl 1040.20024

[Wise 2006] D. T. Wise, "Subgroup separability of the figure 8 knot group", Topology 45:3 (2006), 421-463. MR 2007a:57010 Zbl 1097.20030

Received December 17, 2008. Revised July 9, 2009.

\author{
JASON FOX MANNING \\ DEPARTMENT OF MATHEMATICS \\ UNIVERSITY AT BUFFALO \\ BUFFALO, NY 14260-2900 \\ UNITED STATES \\ j399m@buffalo.edu \\ http://www.math.buffalo.edu/ j399m \\ EduARdo Martínez-PEdRoza \\ DEPARTMENT OF MATHEMATICS AND STATISTICS \\ MCMASTER UNIVERSITY \\ HAMILTON, ON L8S 4K1 \\ CANADA \\ emartinez@math.mcmaster.ca \\ http://www.math.mcmaster.ca/ emartine
}

